\title{
ON THE PROBLEM OF THE SPECIFIC FREQUENCY OF GLOBULAR CLUSTERS
}

\author{
Ikram Uralbaevich Tadjibaev \\ Department of Physics \\ Chirchik State Pedagogical Institute of Tashkent Region \\ 104 A. Temur str., Chirchik, Uzbekistan, 111700 \\ fim.dekanat@cspi.uz
}

\begin{abstract}
In the article, on the basis of observational data the problems of the specific frequency of globular clusters are studied. Possible relationships between them and the absolute stellar magnitude of their host galaxy are considered, where the observational data published in the literature were presented. It should be noted that before us the relationship between the specific frequency and the absolute magnitude is shown as exponential functions. An empirical relationship between the specific frequency and the absolute value of the host galaxy were obtained and showed that the dependence of the specific frequency on the absolute magnitude is not linear, but has a quadratic function. It is also shown that the specific frequency determines the number of globular clusters in a given galaxy relative to our Galaxy. Also in the article, based on the results of studies of the specific frequency, some discussions are presented related to the origin and evolution of globular clusters. The results obtained show that the ratios of the specific frequency to the luminosity of the host galaxy are different. Variations in the specific frequency of elliptical galaxies are associated with variations in the mass-to-luminous flux ratio. This may be due to the fact that the number of globular clusters in spiral galaxies per unit of luminosity of the halo and not of the entire galaxy. Analysis of the observational data shows that the values of the specific frequency of spiral galaxies are 5-6 times less than that of giant elliptical ones. As a result of the results of studies of the specific frequency of the globular clusters, unsolved problems are listed and possible solutions are shown. It is noted that the problem posed will be solved even more accurately if it is considered by the types of galaxies.
\end{abstract}

Keywords: globular clusters, observational data, specific frequency, absolute magnitude, host galaxy.

DOI: $10.21303 / 2461-4262.2021 .001687$

\section{Introduction}

Globular clusters (GC) are the oldest objects in the Universe. With typical masses of about $10^{4}-10^{6}$ Solar masses (corresponding to stellar magnitudes $M_{V}=-5$ to -10 ) and their compact sizes (tidal radii of several parsecs), they are observed in the outer regions of galaxies. After Harris's review publication [1], the study of extragalactic globular clusters grew rapidly. GCs are investigated by various methods. At present, it has become obvious that the main parameters of GC are considered as unique indicators when explaining various astrophysical processes. The results of the analysis of observational data GC are used to explain the origin and evolution of galaxies, the role of dark matter in the structure in the early Universe and their distribution in galaxies. GCs are also valuable objects for theoretical and observational astronomy. It should be noted that the works [2-6] summarize the results of the research on GC and them systems. The specific frequency $S_{N}$, GC of elliptical and spiral galaxies plays an important role in using the properties of GC systems to limit the models of galaxy formation. Giant elliptical galaxies are several times more massive than spiral galaxies. Although the dependence of the specific frequency $S_{N}$ and the luminosity $L$ means that this ratio will depend in some way on the brightness of the compared galaxies, that a larger number of GC around elliptical galaxies re spiral ones is a real effect. Thus, the formation of elliptical galaxies by merging is direct: elliptical galaxies have too many GC that have formed by merging spiral galaxies.

The specific frequency of globular clusters plays an important role in determining masses, luminosities, in the distribution of stars, especially in the study of issues related to their origin and formation. And also, it is possible to study the efficiency of formation of globular clusters using the specific frequency. The specific frequency values can explain various models of the origin of galaxies and their subsystems, i. e. globular clusters.

The specific frequency of GC in galaxies differs between galaxies with different morphological Hubble types and between galaxies with different environments. The specific frequency 
of GC and their correlations with the main physical properties of their host galaxies have already been discussed in various contexts of galaxy formation: displaced formation of globular clusters in a high-density medium, the origin of field and elliptical clusters, and the origin of dwarf elliptical galaxies. The results of numerical and theoretical studies also show that the values of the specific frequency in different galaxies are different.

The paper [7] investigates the tendency between the specific frequency and absolute magnitude for galaxies with a wide range of absolute magnitude values. The authors found that the specific frequency values were higher in dwarf galaxies with the lowest luminosity and similar specific frequency values for giant elliptical galaxies. Specific frequencies appear to have a minimum at intermediate luminosities of galaxies, but very few observational data were available for them. In the article [8] investigated the values of the specific frequency for $\sim 100$ early-type galaxies in the Virgo cluster, confirming the high values of the specific frequency for dwarf and low values of the specific frequency at $M_{V} \sim-19.5$. Thus, it is not yet clear that the dependence of the specific frequency and the absolute value is U-shaped, power-law, logarithmic or bimodal.

Despite this, there are many unresolved issues regarding the specific frequency. Therefore, the aim of this work is to find the relationship between the specific frequency and the absolute magnitude of the host galaxy using the GC observational data.

\section{Analysis of observational data}

Harris and van den Berg [9] first introduced the term specific frequency (frequency of occurrence) of GC in a galaxy (or the number of GC per unit of galaxy luminosity), which is still used today:

$$
S_{N}=N_{G C} \times 10^{0.4\left(M_{V}+15\right)}
$$

where $M_{V}$ - integral absolute value of the galaxy and $N_{G C}$ - total number of GC.

Statistical dependence (1) has been widely used in various models to explain the mechanisms of galaxy formation, for example, the formation of giant elliptical galaxies from the merger of two disk galaxies. The number of GC in the system is determined by doubling the number of GC brighter than the turning point of the luminosity function. This definition has both practical and physical meaning. The weak end of the luminosity function in many cases is determined very poorly, because $\sim 90 \%$ of the mass of the GCS is in the bright part.

Comparison of the specific frequency $S_{N}$ among different types of galaxies will be valid only if all galaxies have the same mass-luminosity ratio. Therefore, the value $T$ was introduced in the work [9], - the number of GC per unit $10^{9}$ of the stellar mass of the galaxy. Since the mass-luminosity ratio for a particular galaxy is not known, it is therefore used as a scaling factor that differs for each type of galaxy. Below let's discuss the results of observations of the specific frequency $S_{N}$. Direct estimate stellar masses for individual galaxies would be one more efficient approach.

As a merger model was proposed, the author of [10] argued that elliptical galaxies could not form as a result of merging spiral galaxies, since spiral galaxies have lower $\mathrm{S}_{\mathrm{N}}$ values than elliptical. In a series of works [11], it was assumed that the problem of specific frequency can be solved if new GC were formed only in the process of merging of two galaxies. If the rate of formation of GC efficiency is high, then as a result of the merger of galaxies, the value of the specific frequency increases. Studies of a young cluster in nearby spiral galaxies show that galaxies with high star formation rates have more young clusters, but it is unlikely that galaxy mergers are more effective in GC formation.

In recent years, several broadband and space-based GC studies have been carried out to estimate the exact value of the specific frequency of a given galaxy. It turned out that it was necessary to revise some of the previously obtained specific frequency results and to compare the new results with the old ones. Because reliable results can be obtained by improved photometry. In some cases, the luminosity of the host galaxy is slightly lower than that of the observed one. For example, the luminosity of the giant elliptical galaxy NGC 1399 in the Fornax clusters was slightly lower than in observed one. Since the specific frequency is related to the luminosity of the host galaxy, it is necessary to correct the value of the specific frequency. The specific frequency for this galaxy was revised and decreased to almost two times, i. e. $\sim 12$ to 5-6. 
The evolutionary history of galaxies located in the central parts of a cluster of galaxies differs from other elliptical galaxies of similar mass. This resulted in a high specific frequency. In addition, galaxies in high-density environments have a higher specific frequency. Many authors argue that high values of the specific frequency in central galaxies arise due to hot gas in the central parts of clusters, and they are not associated with the formation of GC. The properties of elliptical galaxies NGC 4636 may also be consistent from this point of view. Despite its relatively low-density environment, this galaxy has a specific frequency of $\sim 6$, which is typical of the cluster's central galaxies. However, the galaxy NGC 4636 has a dark matter halo that characterizes its massiveness.

Harris in his work [1] shows a monotonic increase in the specific frequency. He thought that with an increase in the mass of the host galaxy, the value of the specific frequency increases, but in fact it is not so. Observations show that the value of the specific frequency also increases towards low luminosities, i. e. also for dwarf galaxies.

It should be noted that in the work [12] the authors were interested in this dependence. Using numerical simulations, they investigated the specific frequency of the GC in order to explain the origin of the U-shaped dependence. The authors find dependence in the form of a power law:

$$
S_{N}(x)=A_{1} \cdot 10^{K_{1} x}+\left(S_{N, t h}-A_{1}\right) \cdot 10^{K_{2} x},
$$

where $x=\left(M_{V}-M_{V, t h}\right) / M_{V, t h}, S_{N, t h}$, equal to value $S_{N}$ at $M_{V}=M_{V, t h}, a A_{1}, K_{1}, K_{2}-$ ratio. By comparing the observed values of the specific frequency with the theoretical one, the authors found the following constants: $M_{V, t h}=-19 .{ }^{m} 5, S_{N, t h}=1.0, A_{1}=0.5, K_{1}=-6.0, K_{2}=4.0$.

Also, the authors found that in early-type galaxies with low mass in the stellar magnitude range $-20^{m}, 5<M_{V}<-16^{m} .0$ specific frequency depends very weakly. If the ratio of the halo mass to the galaxy luminosity is described by the law $M_{\text {halo }} / L \sim M_{\text {halo }}^{\alpha}$, where $\alpha=-1$, then the dependence of the specific frequency on the luminosity is well correlated. Based on these results, the authors analyzed the U-shape of the dependence of the specific frequency on the absolute magnitude of the galaxy.

\section{Results}

As it is seen from equations (1), the specific frequency depends on the absolute value of the host galaxy and the number of GCs in the system. The values of the specific frequency are taken mainly from the following works [13-18]. At Fig. 1 the dependence of the specific frequency on the absolute value of the host galaxy is shown based on the above data. It is seen that the dependence is described most of all by a quadratic equation in the form:

$$
S_{N}=a_{0}+a_{1} M_{V}+a_{2} M_{V}^{2}
$$

where $a_{0}, a_{1}, a_{2}$ - coefficients. Using the least squares method, let's found these coefficients: $a_{0}=133.24$ ( \pm 12.14$), a_{1}=13.62(1.26), a_{2}=0.35$ (0.031). Mean square error or dispersion 4.25 .

It is interesting that a comparison of formula (2), (3) shows that the mean square error is less in the quadratic formula than in the power one (error 5.37). This means that the dependence is described, most of all, by a quadratic formula.

Another interesting fact. Let's introduce the designation: $y=N_{G C} / N_{G C M W}$ is the approximate number of GCs in the system, and $x=\left(0.4 M_{V}\right) / M_{V M W}-1$ is the absolute magnitude relative to our Galaxy. In this case we in formula (1) instead of $N_{G C}$ put $N_{G C} / N_{G C M W}$ (here $N_{G C M W}$ - number GC in our galaxy, i. e. $\left.N_{G C M W}=160\right)$ and $M_{V}+15$ is $M_{V} / M_{V M W}-1\left(M_{V M W}=-21.3\right.$ - the absolute stellar magnitude of our Galaxy) - then the left part shows the approximate number of GC in the system around any galaxies, relative to our Galaxy. Fig. 2 shows this dependence.

Further, using the same observational data, let's perform an approximation with a power function in the form of:

$$
y=A_{1} \cdot 10^{A_{2} x},
$$


and the coefficients were found with the least squares method, which is equal to the following value: $A_{1}=-7.234( \pm 0.008)$ and $A_{2}=-0.36( \pm 0.0004)$. Mean square error or dispersion 0.02 .

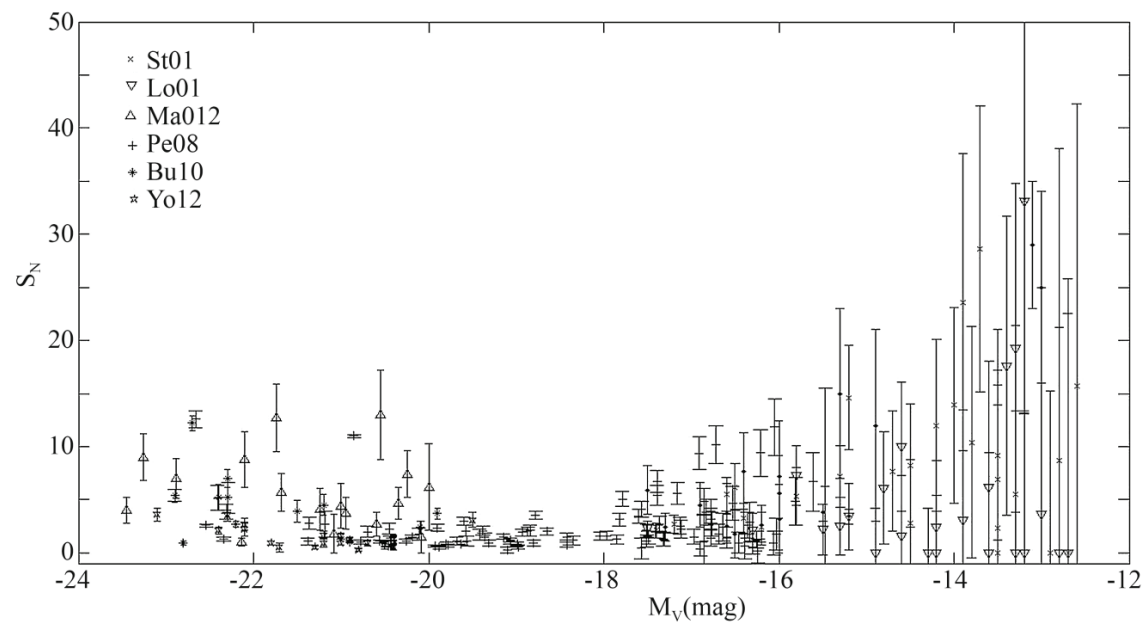

Fig. 1. Dependence of the specific frequency on the absolute value of the host galaxies. Designation: St01 [13], Lo01 [14], Ma02 [15], Pe08 [16], Bu10 [17], Yol2 [18]

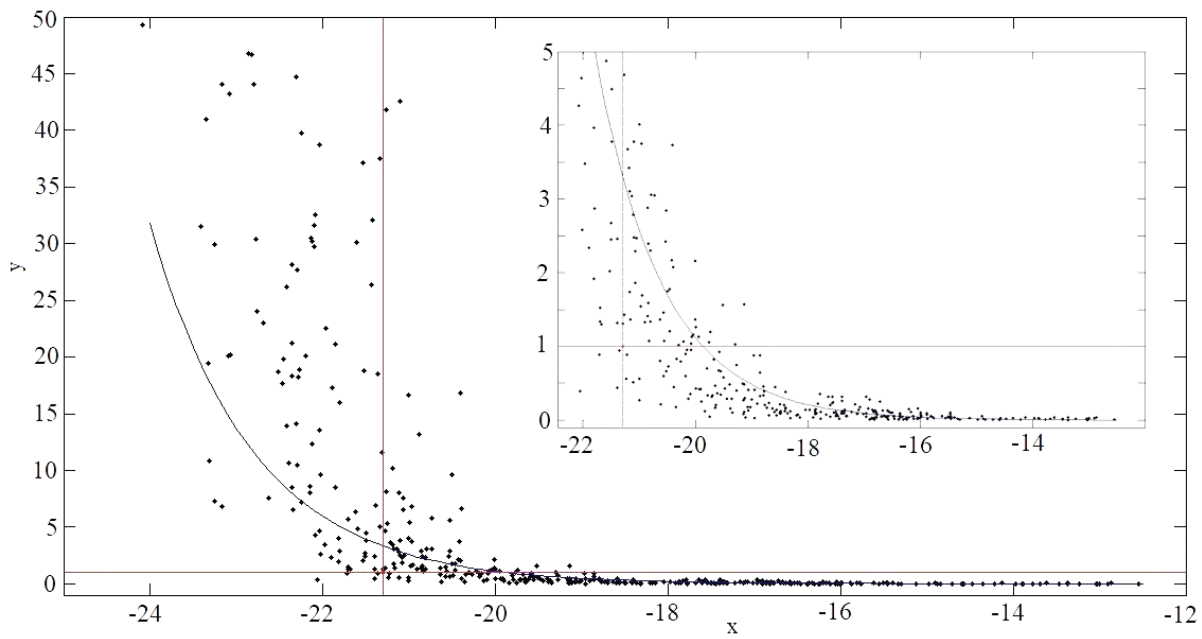

Fig. 2. Dependence of the specific frequency on the absolute value of the host galaxy relative to our Galaxy. The central part is shown separately on a large scale

In Fig. 2 it is shown as continuous lines. Straight lines match values $S_{N}=1$ and $M_{V}=-21.5$, respectively. In the upper panel, the central part of the graph is shown on a large scale.

\section{Discussion of experimental results}

As noted above, the specific frequency of $\mathrm{GC}$ is directly related to their origin and evolution. Here are some discussions in this direction. Since the results obtained show that the ratios of the specific frequency to the luminosity of the host galaxy have differences and they are explained in different ways. Extremely high values of specific frequency for giant $c D$ galaxies, some variations in specific frequency in elliptical galaxies are associated with variations in the mass-to-luminous flux ratio, which increases with increasing luminosity of the host galaxy. Let's note one more reason: this may be the number of GC in spiral galaxies per unit of halo luminosity, not the entire galaxy. It makes more sense to compare halos here because the disks of spiral galaxies are still bright with active star formation. The decrease in specific frequency with increasing luminosity of dwarf galaxies is explained as a result of gas removal during the star formation phase in the 
host galaxy after the formation of GC. Removing the gas reduces the mass and luminosity of the host galaxy, but does not change the number of GC. On the other hand, the correlation between the specific frequency and luminosity concerns the continuous destruction of GC systems long after their formation.

The specific frequency of GC in spiral galaxies is about 5-6 times lower than in elliptical ones, and also 5 times lower in elliptical galaxies compared to giant $c D$ galaxies. This phenomenon is associated with the fact that the result of changes in the ratio of mass to luminosity, especially among elliptical galaxies with increasing luminosity. Variations in the specific frequency of about 2 times seem likely for merging galaxies that form GC during the star formation phase. The efficiency formation of GC per unit of total gas mass is surprisingly constant in different media, because most of the changes in specific frequency can be explained with changes in the ratio of total mass to luminosity. As a result of the research results, it is possible to list the following unresolved questions: why is the efficiency of formation of connected GC constant from place to place and over time? When the efficiency of formation of GC is constant, why does the specific frequency change? Why should the efficiency of formation of GC be constant than the efficiency of the entire star formation? When and how did the red and blue populations of GC in spiral and elliptical galaxies form? Etc. To study such questions, it is necessary to create a huge database of observational data on the specific frequency of GC. It should be noted that the problem posed by us will be solved more accurately if to consider separately by morphological types of galaxies. This requires additional research in this direction.

Analysis of the data shows that it is difficult to determine the number of GCs in a given system based on observations, but if find the values of the specific frequency, then it is easy to determine their number using the formula (3) or (4). Also, the use of the obtained formulas (3), (4) is very easy and is better agreed with the data of observations.

\section{Conclusion}

On the basis of observational data, the problems of specific frequency are studied and it is shown that it depends on the absolute stellar magnitude of the host galaxy in a quadratic form. It is shown that the specific frequency determines the number of GC in a given galaxy relative to our Galaxy. Possible empirical relationships between the specific frequency and the absolute value of the host galaxy have been obtained.

\section{Acknowledgments}

The authors are grateful to professor S. N. Nuritdinov for posing the problems and discussing the results. The work was carried out under the grant OT-F2-13 of the Ministry of Innovative Development of Uzbekistan.

\section{References}

[1] Harris, W. E. (1991). Globular Cluster Systems in Galaxies Beyond the Local Group. Annual Review of Astronomy and Astrophysics, 29 (1), 543-579. doi: https://doi.org/10.1146/annurev.aa.29.090191.002551

[2] Ashman,K.,Zepf, S.(1998). Globular cluster systems. Cambridge University Press. doi: https://doi.org/10.1017/cbo9780511564604

[3] Tadjibaev, I. U., Nuritdinov, S. N., Ganiev, J. M. (2015). Globular Star Cluster Systems Around Galaxies. II. Spiral and Dwarf Galaxies. Astrophysics, 58 (2), 181-192. doi: https://doi.org/10.1007/s10511-015-9374-9

[4] Tadjibaev, I. U., Nuritdinov, S. N., Muminov, A. A. (2017). Non-Linear Cosmology of Globular Cluster Systems around Galaxies. Ukrainian Journal of Physics, 62 (12), 1057-1064. doi: https://doi.org/10.15407/ujpe62.12.1057

[5] Tadjibaev, I. U., Nuritdinov, S. N. (2019). Can Globular Clusters in the Galaxy Be Classified by the Velocity Anisotropy Parameter? Ukrainian Journal of Physics, 64 (4), 271. doi: https://doi.org/10.15407/ujpe64.4.271

[6] Tadjibaev, I. U. (2020). On the theory of the origin of global cluster systems around the galaxies. Eurasian Union Scientists, 2 (7 (76)), 59-64. doi: https://doi.org/10.31618/esu.2413-9335.2020.2.76.899

[7] Durrell, P. R., Harris, W. E., Geisler, D., Pudritz, R. E. (1996). Globular Cluster Systems in Dwarf Elliptical Galaxies. II. The Virgo Cluster. The Astronomical Journal, 112, 972. doi: https://doi.org/10.1086/118071

[8] Forbes, D. A. (2005). Bimodal Galaxies and Bimodality in Globular Cluster Systems. The Astrophysical Journal, 635 (2), L137-L140. doi: https://doi.org/10.1086/499563 
[9] Harris, W. E., van den Bergh, S. (1981). Globular clusters in galaxies beyond the local group. I - New cluster systems in selected northern ellipticals. The Astronomical Journal, 86, 1627. doi: https://doi.org/10.1086/113047

[10] Van den Bergh, S. (1982). Globular clusters and galaxy mergers. Publications of the Astronomical Society of the Pacific, 94, 459. doi: https://doi.org/10.1086/131007

[11] Ashman, K. M., Zepf, S. E. (1992). The formation of globular clusters in merging and interacting galaxies. The Astrophysical Journal, 384, 50. doi: https://doi.org/10.1086/170850

[12] Bekki, K., Yahagi, H., Forbes, D. A. (2006). The U-shaped Distribution of Globular Cluster-specific Frequencies in a Biased Globular Cluster Formation Scenario. The Astrophysical Journal, 645 (1), L29-L32. doi: https://doi.org/10.1086/505905

[13] Stiavelli, M., Miller, B. W., Ferguson, H. C., Mack, J., Whitmore, B. C., Lotz, J. M. (2001). The Nuclear Cusp Slopes of Dwarf Elliptical Galaxies. The Astronomical Journal, 121 (3), 1385-1394. doi: https://doi.org/10.1086/319425

[14] Lotz, J. M., Telford, R., Ferguson, H. C., Miller, B. W., Stiavelli, M., Mack, J. (2001). Dynamical Friction in dE Globular Cluster Systems. The Astrophysical Journal, 552 (2), 572-581. doi: https://doi.org/10.1086/320545

[15] Marin-Franch, A., Aparicio, A. (2002). Globular Cluster Systems in Elliptical Galaxies of Coma. The Astrophysical Journal, 568 (1), 174-189. doi: https://doi.org/10.1086/338839

[16] Peng, E. W., Jordán, A., Côté, P., Takamiya, M., West, M. J., Blakeslee, J. P. et. al. (2008). The ACS Virgo Cluster Survey. XV. The Formation Efficiencies of Globular Clusters in Early-Type Galaxies: The Effects of Mass and Environment. The Astrophysical Journal, 681 (1), 197-224. doi: https://doi.org/10.1086/587951

[17] Burkert, A., Tremaine, S. (2010). A correlation between central supermassive black holes and the globular cluster systems of early-type galaxies. The Astrophysical Journal, 720 (1), 516-521. doi: https://doi.org/10.1088/0004-637x/720/1/516

[18] Young, M. D., Dowell, J. L., Rhode, K. L. (2012). Globular cluster systems of spiral and S0 galaxies: Results from WIYN imaging of NGC 1023, NGC 1055, NGC 7332, and NGC 7339. The Astronomical Journal, 144 (4), 103. doi: https:/doi.org/ $10.1088 / 0004-6256 / 144 / 4 / 103$

Received date 17.09.2020

(C) The Author(s) 2021

Accepted date 13.02.2021

This is an open access article under the CC BY license

Published date 31.03.2021 (http://creativecommons.org/licenses/by/4.0).

How to cite: Tadjibaev, I. U. (2021). On the problem of the specific frequency of globular clusters. EUREKA: Physics and Engineering, 2, 137-142. doi: https://doi.org/10.21303/2461-4262.2021.001687 